\title{
NONTHERMAL ORIGIN OF X-RAYS FROM ROTATION-POWERED NEUTRON STARS
}

\author{
K. S. Cheng, ${ }^{1}$ J. GIL, ${ }^{2}$ AND L. ZHANG ${ }^{1}$ \\ Received 1997 May 7; accepted 1997 November 12; published 1998 January 6
}

\begin{abstract}
We propose that the bulk of X-rays observed in pulsars results from the synchrotron radiation of $e^{ \pm}$pairs created in strong pulsar magnetic field near the neutron star surface by curvature photons emitted by charged particles on their way from the outer gap to the neutron star surface. The characteristic energy of these curvature photons is completely determined by the size of the outer gap, which is self-consistently limited by thermal Xrays from the neutron star surface heated by the backflow electrons/positrons from the outer gap. It is this limitation of the outer gap size (potential drop) that constrains the luminosity $L_{\mathrm{X}}$ of the synchrotron X-rays emitted by a pulsar to about $0.1 \%$ of its spin-down power $L_{\mathrm{sd}}$.
\end{abstract}

Subject headings: pulsars: general — radiation mechanisms: nonthermal — radiation mechanisms: thermal — X-rays: stars

\section{INTRODUCTION}

The X-ray emission from 27 rotation powered pulsars have been detected so far by ROSAT and ASCA. The observed emission properties in the soft X-ray band have been recently reviewed by Becker \& Trümper (1997). The most remarkable effect reported in this review is an apparently linear relationship between the X-ray luminosity $L_{\mathrm{X}}$ for all rotation-powered pulsars detected by ROSAT and the spin-down luminosity $L_{\mathrm{sd}}$. A very narrow distribution of $L_{\mathrm{X}}$ around $10^{-3} L_{\mathrm{sd}}$ extends over 8 orders of magnitude. Seward \& Wang (1988) were the first to point out a possibility of such correlation for the Einstein data and Ögelman (1995) has advocated for a strong effect using a smaller sample of the ROSAT data.

There are many possible mechanisms to explain the X-ray emission from pulsars, including the internal frictional dissipation between the stellar crust and the crustal superfluid (Alpar et al. 1984; Shibazaki \& Lamb 1988; Cheng et al. 1992), polar gap heating (Arons 1981; Harding, Ozernoy, \& Usov 1993), outer gap heating (Cheng 1987; Halpern \& Ruderman 1993), accretion from interstellar medium (Paczyński 1990), accretion of the white dwarf companion onto the neutron star (Edelstein, Foster, \& Bowyer 1995), pulsar wind nebular emission (Arons \& Tavani 1993), monopole-catalyzed nucleon decay inside the neutron stars (Freese, Turner, \& Schramm 1983), etc. However, Becker \& Trümper (1997) argued that the surprisingly narrow correlation between $L_{\mathrm{X}}$ and $L_{\mathrm{sd}}$, holding for both canonical and millisecond pulsars, strongly suggests that the bulk of the observed X-rays is emitted at the expense of rotational energy of a neutron star, and therefore, it has to be of a nonthermal magnetospheric origin. In fact, there is no reason why any kind of thermal emission should reflect almost exactly $0.1 \%$ of the rotational energy available. In this Letter we propose a model that explains the linear relationship $L_{\mathrm{X}} \approx 10^{-3} L_{\mathrm{sd}}$ by means of magnetospheric synchrotron radiation of secondary $e^{ \pm}$pairs produced by high-energy curvature photons in the strong pulsar magnetic field near the neutron star surface.

\footnotetext{
${ }^{1}$ Department of Physics, University of Hong Kong, Pokfulam Road, Hong Kong.

2 Astronomy Center, Pedagogical University, Lubuska 2, 65265 Zielona Gora, Poland.
}

\section{THE MODEL}

High-energy $\gamma$-ray emission can be explained in terms of either polar cap models (see, e.g., Harding 1981; Dermer \& Sturner 1994; Rudak \& Dyks 1998) or outer gap models (see, e.g., Cheng, Ho, \& Ruderman 1986a, 1986b; Ho 1989; Cheng \& Ding 1994; Cheng \& Wei 1995; Romani 1996). In this Letter, we use a newly developed outer gap model proposed by Zhang \& Cheng (1997). In their model the characteristic photon energy is completely determined by the size of the outer gap $(f)$ and is given by $E_{\gamma}^{\mathrm{sc}}(f) \approx 2 \times 10^{8} f^{3 / 2} B_{12}^{3 / 4} P^{-7 / 4} \mathrm{eV}$, where $P$ is the pulsar period in units of seconds, $B_{12}$ is the dipolar magnetic field in units of $10^{12} \mathrm{G}$, and the radius of the neutron star $(R)$ is assumed to be $10^{6} \mathrm{~cm}$. Half of the primary $e^{ \pm}$pairs in the outer gap will move toward the star and loss their energy via the curvature radiation. The return particle flux can be approximated by $\dot{N}_{e^{ \pm}} \approx \frac{1}{2} f \dot{N}_{\mathrm{GJ}}$, where $\dot{N}_{\mathrm{GJ}}$ is the Goldreich-Julian particle flux (Goldreich \& Julian 1969). Although most of the energy of the primary particles will loss on the way to the star via curvature radiation, about $10.6 P^{1 / 3}$ ergs per particle will still remain and finally deposit on the stellar surface. This energy will be emitted in terms of X-rays from the stellar surface (Halpern \& Ruderman 1993). The characteristic energy of Xrays is given by $E_{\mathrm{X}}^{h} \approx 3 k T \approx 1.2 \times 10^{3} P^{-1 / 6} B_{12}^{1 / 4} \mathrm{eV}$. The $\mathrm{keV}$ $\mathrm{X}$-rays from a hot polar cap will be reflected back to the stellar surface due to the cyclotron resonance scattering if there is large density of magnetic produced $e^{ \pm}$pairs near the neutron star surface (Halpern \& Ruderman 1993), and eventually reemit softer thermal X-rays with characteristic energy $E_{\mathrm{X}}^{s} \approx$ $0.1 f^{1 / 4} P^{-1 / 4} E_{\mathrm{X}}^{h}$.

Despite the $\mathrm{X}$-ray photon density is very low, every pair produced by X-ray and curvature photons collision can emit $10^{5}$ photons in the gap. Such huge multiplicity can produce sufficient number of $e^{ \pm}$pairs to sustain the gap as long as the center of mass energy of X-ray and curvature photon is higher than the threshold energy of the electron/positron pair production, i.e., $E_{\mathrm{X}} E_{\gamma}^{\mathrm{sc}}(f) \geq\left(m_{e} c^{2}\right)^{2}$. From the condition for the photon-photon pair production, the size of the outer gap limited by the soft thermal X-rays from the neutron star surface can be determined as

$$
f_{s}=5.5 P^{26 / 21} B_{12}^{-4 / 7} \text {. }
$$

It should be emphasized that $f_{s} \leq 1$. If $f_{s}>1$, it means that there is not sufficient $e^{ \pm}$pairs near the neutron star surface and then 
the keV X-rays from a hot polar cap will escape from the star. In this case, the size of the outer gap is limited by the hard Xrays and is given by

$$
f_{h} \approx 1.6 P^{23 / 18} B_{12}^{-2 / 3} .
$$

Obviously, if both $f_{s}$ and $f_{h}$ are greater than 1 , then a selfsustained outer gap does not exist. Any fluctuation of the gap can be stabilized by the process described above. In fact, the increase of gap size will increase the X-ray flux and the overproduced pairs can reduce the gap size. Similarly, the decrease of the gap size will produce insufficient pairs and results in the increase of the gap size (see also Cheng 1994). The primary electrons/positrons leaving the outer gap emit curvature photons with typical energy

$$
E_{\gamma}=(3 / 2)(c / s) \hbar \gamma(x)^{3},
$$

where $x=s / R_{L}, s$ is the local radius of curvature, $R_{L}=$ $c p / 2 \pi$ is the light cylinder radius, and $\gamma(x) \approx 2 \times$ $10^{7} f^{1 / 2} B_{12}^{1 / 4} P^{-1 / 4} x^{-3 / 4}$ (eq. [49] of Zhang \& Cheng 1997). The value of $x$ evaluated at the position $\left(x_{\min }\right)$, where the primary electrons/positrons just leave the outer gap, depends sensitively on the inclination angle $\chi$. Zhang \& Cheng (1997) have estimated that $x_{\min }$ could be as small as 0.3 . This value of $x_{\min }$ yields $E_{\gamma} \sim 25.6 \mathrm{GeV}$ for Geminga, which is consistent with the estimation by Halpern \& Ruderman (1993). We want to emphasize that the curvature photon energy emitted by the backflow electron/positrons is higher than that of the average curvature photon energy because the radius of curvature at the inner boundary of the outer gap can be smaller than that of the average value by a factor of 3 . These photons will be converted into the secondary $e^{ \pm}$pairs by the neutron star magnetic field if the pair production condition in strong magnetic field is satisfied (Erber 1966; Ruderman \& Sutherland 1975), i.e.,

$$
\frac{E_{\gamma}}{2 m_{e} c^{2}} \frac{B\left(r_{s}\right)}{B_{g}} \approx \frac{1}{15},
$$

where $B\left(r_{s}\right)$ is local magnetic field at position of $r_{s}$ and $B_{g}=$ $m_{e}^{2} c^{3} / e \hbar=4.4 \times 10^{13} \mathrm{G}$. From this condition and assuming that the local field is a dipole field, then the distance to the star of the first generation of $e^{ \pm}$pairs can be given by

$$
\frac{r_{s}}{R}=\left(\frac{15 E_{\gamma} B_{s}}{2 m c^{2} B_{g}}\right)^{1 / 3}
$$

where $B_{s}$ is the surface magnetic field. These secondary $e^{ \pm}$ pairs will lose their energy via synchrotron radiation with the following typical energy

$$
E_{\mathrm{syn}}=\frac{3}{2}\left(\frac{E_{\gamma}}{2 m_{e} c^{2}}\right)^{2} \hbar \frac{e B\left(r_{s}\right)}{m_{e} c}=\frac{E_{\gamma}}{20},
$$

where we have used equation (4) to simplify equation (6). For the parameters of Geminga, i.e., $P=0.237 \mathrm{~s}, B_{12}=1.6$, and $x_{\min }=0.3$, equation (3) gives the energy of the curvature photons $E_{\gamma}=25.6 \mathrm{GeV}$, equation (5) gives the position of the first generation of $e^{ \pm}$pairs $r_{s} / R=24.0$, and equation (6) gives the energy of first generation of synchrotron radiation $E_{\mathrm{syn}} \sim$ $1.28 \mathrm{GeV}$. Since these synchrotron photons are emitted toward the star, they will encounter stronger magnetic field and convert into $e^{ \pm}$pairs, which again radiate their energy via synchrotron radiation. We can derive a general formula for the typical energy of each generation of $e^{ \pm}$pairs. From equation (4), we get

$$
\gamma_{i}=\frac{B_{g}}{B_{s} R^{3}} \frac{r_{i}^{3}}{15} \text {, }
$$

where $\gamma_{i}$ and $r_{i}$ are the characteristic Lorentz factor and the radial position of the $i$ th generation $e^{ \pm}$pairs when they are just created. Then the typical energy of the $i$ th generation synchrotron photons is given by $\left(E_{\mathrm{syn}}\right)_{i}=\frac{3}{2} \gamma_{i}^{2} \hbar e B\left(r_{i}\right) / m c$. These photons will become pairs at $r_{i+1}$ with the typical Lorentz factor

$$
\gamma_{i+1}=\frac{3}{2} \frac{\hbar}{2 m_{e} c^{2}} \frac{e B_{s}}{m_{e} c}\left(\frac{r_{i}}{R}\right)^{-3}\left(\frac{B_{g} r_{i}^{3}}{15 B_{s} R^{3}}\right)^{2},
$$

and satisfying equation (4) yields

$$
\gamma_{i+1}=\frac{B_{g} r_{i+1}^{3}}{15 B_{s} R^{3}}
$$

We can easily show that

$$
\frac{r_{i+1}}{r_{i}}=\left(\frac{1}{20}\right)^{1 / 3}
$$

and

$$
\frac{\gamma_{i+1}}{\gamma_{i}}=\left(\frac{r_{i+1}}{r_{i}}\right)^{3}
$$

Therefore, each new generation of $e^{ \pm}$pairs will reduce their energy by a factor of $\sim 0.05$ and comes closer to the star by a factor of $\sim 0.37$. In case of Geminga, the pairs start with energy $\sim 1.28 \mathrm{GeV}$. It takes about two generations to reduce their energy $1.28 \mathrm{GeV} \times(0.05)^{2} \mathrm{MeV} \sim 3.2 \mathrm{MeV}$, and the pair production processes stop at $r_{f} / R \sim\left(r_{s} / R\right)(1 / 20)^{2 / 3} \sim 3.3$. The synchrotron spectral index starts with -1.5 and evolves to become -1.9. We argue that an electromagnetic cascade will take place until the energy of synchrotron photons is $\sim 1 \mathrm{MeV}$ and the spectral index $\sim-2$. The total number of secondary $e^{ \pm}$pairs created in the electromagnetic cascade is given by Zhang \& Cheng (1997) $\dot{N}^{(2)}=\left(\dot{N}^{(1)} \gamma m c^{2} / \mathrm{MeV}\right)\left(r_{s} / f r_{i}\right)^{2} \mathrm{~s}^{-1}$, where $\dot{N}^{(1)} \approx f \dot{N}_{\mathrm{GJ}}$ and $\dot{N}_{\mathrm{GJ}}=2.7 \times 10^{30} B_{12} P^{-2} \mathrm{~s}^{-1}$ is the GoldreichJulian particle flux, $r_{i} \sim 4 R_{L} / 9 \tan ^{2} \chi$ is the distance to the inner boundary of the outer gap. Furthermore, the synchrotron spectrum is given by

$$
\frac{d N\left(E_{\mathrm{X}}\right)}{d E_{\mathrm{X}}}=A\left(\frac{E_{\mathrm{X}}}{\mathrm{MeV}}\right)^{-2}, \hbar \frac{e B\left(r_{f}\right)}{m_{e} c} \leq E_{\mathrm{X}} \leq 1 \mathrm{MeV},
$$

where $r_{f}$ is the position where the electromagnetic cascade stops and

$$
A \approx \frac{\dot{N}_{e^{ \pm}}^{(2)}}{\ln \left\{1 \mathrm{MeV} / \hbar\left[e B\left(r_{f}\right) / m_{e} c\right]\right\} \quad \mathrm{MeV}} .
$$

For Geminga, $r_{f} \approx 3.3 R$ and $\hbar\left[e B\left(r_{f}\right) / m_{e} c\right] \sim 489 \mathrm{eV}$. Since most of the energy of secondary $e^{ \pm}$is radiated away in form of synchrotron X-rays, we have the X-ray luminosity $L_{\mathrm{X}} \approx$ $\mathrm{MeV} \cdot \dot{N}^{(2)}$. The pulsar spin-down luminosity is $L_{\mathrm{sd}}=3.8 \times$ 


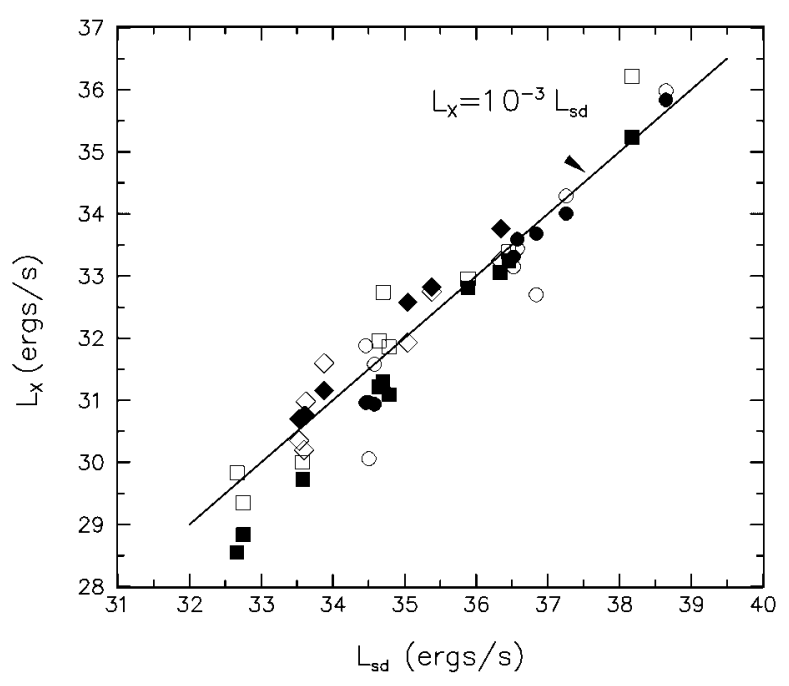

FIG. 1.-Plot of the X-ray pulsar luminosity vs. the spin-down luminosity for 25 pulsars. The data points are represented by open symbols (after Becker \& Trümper), while theoretical values are represented by filled symbols. Pulsars with detected X-ray emission are marked by circles, millisecond pulsars are marked by diamonds and canonical pulsars with no $\gamma$-rays detected are marked by squares.

$10^{31} B_{12}^{2} P^{-4}$ ergs $\mathrm{s}^{-1}$. Thus, writing $r_{s} / r_{i}=(9 / 4)\left(r_{s} / R_{L}\right) \tan ^{2} \chi$ and using $\dot{N}^{(2)}$, we obtain

$$
L_{\mathrm{X}} \approx 8.8 \times 10^{-6} \tan ^{4}(\chi) f^{1 / 2} B_{12}^{5 / 12} P^{-17 / 12} L_{\mathrm{sd}} .
$$

If we take $f=f_{s}$, which is less than unity for most X-ray pulsars, then we have

$$
L_{\mathrm{X}} \approx 5.5 \times 10^{-4}\left(\frac{\tan \chi}{\tan 55^{\circ}}\right)^{4} B_{12}^{0.13} P_{-1}^{-0.80} L_{\mathrm{sd}}
$$

and $L_{\mathrm{X}} \approx 10^{-3} L_{\mathrm{sd}}$ for the Crab pulsar. In the case of millisecond pulsars, for which the light cylinder is much closer to the star than that in canonical pulsars we have to use $r_{s} \approx R$ (the much stronger multipole surface magnetic field will allow the copious pair production there) and

$$
L_{\mathrm{X}}^{\mathrm{msec}} \sim 10^{-3}\left(\frac{\tan \chi}{\tan 55^{\circ}}\right)^{4} B_{9}^{1 / 12} P_{-2}^{-7 / 9} L_{\mathrm{sd}} .
$$

For $P=0.004 \mathrm{~s}, B_{12}=0.0005$, and $\chi=55^{\circ}$ one obtains $L_{\mathrm{X}}^{\mathrm{msec}}=0.002 L_{\mathrm{sd}}$.

\section{APPLICATIONS}

The results of calculations corresponding to $\chi=55^{\circ}$ in equations (15) and (16) for 25 pulsars from Table 1 in Becker \& Trümper (1997) are presented in Figure 1. The data points from Becker \& Trümper (1997) (their Fig. 4 and Table 1) are reproduced by open symbols, and corresponding theoretical values are represented by filled symbols. Pulsars with detected $\gamma$ ray emission are marked by circles, diamonds represent millisecond pulsars and squares correspond to canonical pulsars with no $\gamma$-ray emission detected. In Table 1 , the typical parameters and expected values for 25 pulsars are given.

As one can see from Figure 1, both linear relationship and scatter of $L_{\mathrm{X}}$ around $10^{-3} L_{\mathrm{sd}}$-values are reproduced by our model well. According to equations (15) and (16), the scatter
TABLE 1

\begin{tabular}{|c|c|c|c|c|c|c|c|}
\hline Pulsar & $\begin{array}{c}P \\
(\mathrm{~ms})\end{array}$ & $\begin{array}{l}\log B \\
(\mathrm{G})\end{array}$ & $\begin{array}{c}d \\
(\mathrm{kpc})\end{array}$ & $f_{s}$ & $f_{h}$ & $\begin{array}{c}\log L_{\mathrm{x}}^{\text {obs }} \\
\left(\operatorname{ergs~s}^{-1}\right)\end{array}$ & $\begin{array}{c}\log L_{\mathrm{X}}^{\mathrm{th}} \\
\left(\operatorname{ergs~s}^{-1}\right)\end{array}$ \\
\hline $\mathrm{B} 0531+21$ & 33.40 & 12.58 & 2.00 & 0.04 & 0.01 & 35.98 & 35.84 \\
\hline B0833-45 & 89.29 & 12.53 & 0.50 & 0.14 & 0.04 & 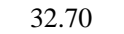 & 33.72 \\
\hline B $0633+17$ & 237.09 & 12.21 & 0.16 & 0.70 & 0.20 & 31.22 & 30.97 \\
\hline B1706-44 & 102.45 & 12.49 & 1.82 & 0.17 & 0.05 & 33.15 & 33.31 \\
\hline B1509-58 & 150.23 & 13.19 & 4.40 & 0.11 & 0.03 & 34.29 & 34.00 \\
\hline B $1951+32$ & 39.53 & 11.69 & 2.50 & 0.15 & 0.04 & 3. & 33.59 \\
\hline B1259-63 & 47.76 & 11.51 & 2.00 & 0.24 & 0.07 & 32.95 & 32.81 \\
\hline B1823-13 & 101.45 & 12.45 & 4.12 & 0.18 & 0.05 & 33.39 & 33.25 \\
\hline B1800-21 & 133.61 & 12.63 & 3.94 & 0.20 & 0.06 & 306 & 33.06 \\
\hline B1929+10 & 226.51 & 11.71 & 0.17 & 1.28 & 0.37 & 3 & $29.74^{\mathrm{a}}$ \\
\hline J0437-47 & 5.75 & 8.54 & 0.18 & 0.88 & 0.25 & 30. & 30.76 \\
\hline B1821-24 & 3.05 & 9.35 & 5.50 & 0.14 & 0.04 & 33.24 & 33.76 \\
\hline B $0656+14$ & 384.87 & 12.67 & 0.76 & 0.70 & 0.20 & 33.00 & 30.94 \\
\hline $40-69$ & 50.37 & 12.70 & 49.40 & 0.05 & 0.02 & & 35.24 \\
\hline $\mathrm{J} 2124-33$ & 4.93 & 8.36 & 0.25 & 0.92 & 0.27 & 30. & 30.70 \\
\hline B1957+20 & 1.60 & 8.14 & 1.53 & 0.30 & 0.09 & 31.93 & 32.58 \\
\hline B $0950+08$ & 253.06 & 11.39 & 0.12 & 2.24 & 0.65 & 29.35 & $28.84^{\mathrm{a}}$ \\
\hline $\mathrm{J} 0538+28$ & 143.15 & 11.87 & 1.50 & 0.59 & 0.17 & 32.74 & 31.29 \\
\hline $\mathrm{J} 1012+53$ & 5.25 & 8.45 & 0.52 & 0.88 & 0.26 & 30.20 & 30.76 \\
\hline B1055-52 & 197.10 & 12.03 & 1.53 & 0.71 & 0.21 & 33.8 & 30.96 \\
\hline B $0355+54$ & 156.38 & 11.92 & 2.07 & 0.61 & 0.18 & 31.96 & 31.22 \\
\hline B2334+61 & 495.24 & 12.99 & 2.46 & 0.63 & 0.18 & 31.86 & 31.09 \\
\hline $\mathrm{J} 0218+42$ & 2.32 & 8.63 & 5.70 & 0.25 & 0.07 & 32.75 & 32.83 \\
\hline B082 & 530.66 & 11.99 & 0.38 & 2.54 & 0.74 & 29.83 & $28.55^{\mathrm{a}}$ \\
\hline $\mathrm{J} 0751+18$. & 3.47 & 8.23 & 2.02 & 0.71 & 0.21 & 31.60 & 31.16 \\
\hline
\end{tabular}

Comparison of ObServed Luminosities With Those OF X-RAYS From PULSARS

${ }^{a}$ The values of $L_{\mathrm{x}}^{\text {th }}$ for PSR B1929+10, B0950+08, and B0823+26 are estimated by using eqs. (2) and (14).

is due to a numerical factor depending on $P$ and $B$, which should be largest for small $P$ - and $B$-values. Therefore, from theoretical point of view, discrepancies between the observed and the model values of $L_{\mathrm{X}}$ result from the actual value of the inclination angle, which was set to $\chi=55^{\circ}$ in the model calculations. The largest discrepancy is less than a factor of 100, which is PSR 1055-52 (in fact, this pulsar is likely a orthogonal rotator interpreted by the radio observation; Lyne \& Manchester 1988). Since $L_{X}$ depends on the fourth power of tangent of the inclination angle $\chi$, this discrepancy can be explained easily by about $20^{\circ}$ deviation of the actual inclination $\chi$ angle from $55^{\circ}$. On the other hand many theoretical values (filled symbols) lie close to the observed values (open symbols), meaning that the actual inclination angle $\chi \approx 55^{\circ}$ in these pulsars. However, our intrinsic scatter of $L_{\mathrm{X}}$-values around $10^{-3} L_{\mathrm{sd}}$-values seems a little bit small, regarding large uncertainties in the pulsar distance, the absorption column and the moment of inertia. Therefore, the actual distribution of inclination angles is probably broader, which would automatically imply wider scatter in the X-ray luminosities.

In order to apply our model to the millisecond pulsars, we had to assume that the surface magnetic field, which extends to at least one stellar radius $R=10^{6} \mathrm{~cm}$ from the surface, has a multipolar structure with a much stronger $B$ than one can conclude from the magnetic braking law. This is important for at least two reasons. First, a canonical magnetic field of millisecond pulsars is too weak for the resonant scattering of hard $\mathrm{X}$-rays to occur (and therefore the size of the outer gap would be limited by hard thermal X-rays coming directly from the hot spot near the polar cap). Second, and much more importantly, such a low canonical magnetic field would not allow a magnetic pair production cascade even at $r \approx R$, which is the critical mechanism for our X-ray emission model. The existence of much stronger multipole components of magnetic field near the polar cap have been suggested by many authors for 
different reasons (Sturrock 1971; Ruderman \& Sutherland 1975; Bulik et al 1992; Gil \& Krawczyk 1997). Perhaps the most important reason is a possibility of a cascade pair production in strong and curved magnetic field of an inner gap in radio pulsars. Here we provide an additional evidence of a multipolar surface magnetic field based on the observed X-ray emission of the millisecond pulsars.

\section{SUMMARY AND DISCUSSION}

We developed a model of nonthermal X-ray emission from rotation-powered neutron stars based on the self-consistent thick outer gap model (Zhang \& Cheng 1997). The observed $\mathrm{X}$-rays result from the synchrotron radiation of $e^{ \pm}$pairs created in strong pulsar magnetic field by curvature photons emitted by charged particles backflowing from the outer gap to the neutron star surface. The characteristic energy of that curvature photons is completely determined by the size of the outer gap, which is self-consistently limited by thermal X-rays from the neutron star surface heated by the backflow electrons/positrons accelerated in the outer gap (Zhang \& Cheng 1997). It is this limitation of the outer gap size (potential drop) expressed in equation (1) or equation (2), which constraints the luminosity $L_{\mathrm{X}}$ of the synchrotron X-ray emitted by a pulsar to about $0.1 \%$ of its spin-down power $L_{\text {sd }}$, as observed. Our model explains successfully not only a linear relationship $L_{\mathrm{X}} \propto L_{\mathrm{sd}}$ extending over 8 orders of magnitude, but also a narrow scatter around $10^{-3} L_{\mathrm{sd}}$-values.

Finally, we want to make two remarks. (1) Whether the cascade can be developed sensitively depends on the inclination angle. More detailed comparison between model spectrum and the observed data is necessary. (2) The electromagnetic cascade development in the case of millisecond pulsars is not clear because we have assumed that a much stronger multipole field exists on the surface of millisecond pulsar. However, the actual structure of the multipole field is not known. Further investigation on this subject is necessary.

We thank W. Becker, B. Rudak, and an anonymous referee for interesting discussion and useful comments, and E. Gil for technical assistance. This Letter is partially supported by RGC grant of Hong Kong government and grant KBN 2 P03D 015 12 of the Polish State Committee for Scientific Research.

\section{REFERENCES}

Alpar, M. A., Anderson, P. W., Pines, D., \& Shaham, J. 1984, ApJ, 278, 791

Arons, J. 1981, ApJ, 248, 1099

Arons, J., \& Tavani, M. 1993, ApJ, 403, 249

Becker, W., \& Trümper, J. 1997, A\&A, 326, 652

Bulik, T., Mesharos, P., Woo, J. W., Nagase, F., \& Makishime, K. 1992, ApJ, 395,564

Cheng, K. S. 1987, ApJ, 321, 805

1994, in Proc. Toward a Major Atmospheric Cherenkov Detector III

for TeV Astro/Particle Physics, ed. T. Kifune (Tokyo: Universal Academy Press), 25

Cheng, K. S., Chau, W. Y., Zhang, J. L., \& Chau, H. F. 1992, ApJ, 396, 135

Cheng, K. S., \& Ding, W. K. Y. 1994, ApJ, 431, 724

Cheng, K. S., Ho, C., \& Ruderman, M. A. 1986a, ApJ, 300, 500 1986b, ApJ, 300, 522

Cheng, K. S., \& Wei, D. M. 1995, ApJ, 448, 281

Dermer, C. D., \& Sturner, S. J. 1994, ApJ, 420, L75

Edelstein, J., Foster, R. S., \& Bowyer, S. 1995, ApJ, 454, 442

Erber, T. 1966, Rev. Mod. Phys., 38, 626
Freese, K., Turner, M., \& Schramm, D. N. 1983, Phys. Rev. Lett., 51, 1625

Gil, J., \& Krawczyk, A. 1997, MNRAS, 285, 566

Goldreich, P., \& Julian, W. H. 1969, ApJ, 157, 869

Halpern, J. P., \& Ruderman, M. A. 1993, ApJ, 415, 286

Harding, A. K. 1981, ApJ, 245, 267

Harding, A. K., Ozernoy, L. M., \& Usov, V. V. 1993, MNRAS, 265, 921

Ho, C. 1989, ApJ, 342, 369

Lyne, A. G., \& Manchester, R. N. 1988, MNRAS, 234, 477

Ögelman, H. 1995, in The Lives of Neutron Stars, ed. A. Alpar, U. Kilizoglu, \& J. Van Paradijs (Dordrecht: Kluwer), 101

Paczyński, B. 1990, ApJ, 348, 485

Romani, W. R. 1996, ApJ, 470, 469

Rudak, B., \& Dyks, J. 1998, MNRAS, in press

Ruderman, M. A., \& Sutherland, P. 1975, ApJ, 196, 57

Seward, F. D., \& Wang, Z. R. 1988, ApJ, 332, 199

Shibazaki, N., \& Lamb, F. 1988, ApJ, 346, 808

Sturrock, P. A. 1971, ApJ, 164, 529

Zhang, L., \& Cheng, K. S. 1997, ApJ, 487, 370 